\title{
Reviewed approach to defining the Active Interlock Envelope for Front End ray tracing
}

\author{
S. Seletskiy, T. Shaftan
}

October 2015

NSLS II, Accelerator Division

Brookhaven National Laboratory

\author{
U.S. Department of Energy \\ DOE - Office of Basic Energy Science
}

\footnotetext{
Notice: This manuscript has been authored by employees of Brookhaven Science Associates, LLC under Contract No. DE-SC0012704 with the U.S. Department of Energy. The publisher by accepting the manuscript for publication acknowledges that the United States Government retains a non-exclusive, paid-up, irrevocable, world-wide license to publish or reproduce the published form of this manuscript, or allow others to do so, for United States Government purposes.
} 


\section{DISCLAIMER}

This report was prepared as an account of work sponsored by an agency of the United States Government. Neither the United States Government nor any agency thereof, nor any of their employees, nor any of their contractors, subcontractors, or their employees, makes any warranty, express or implied, or assumes any legal liability or responsibility for the accuracy, completeness, or any third party's use or the results of such use of any information, apparatus, product, or process disclosed, or represents that its use would not infringe privately owned rights. Reference herein to any specific commercial product, process, or service by trade name, trademark, manufacturer, or otherwise, does not necessarily constitute or imply its endorsement, recommendation, or favoring by the United States Government or any agency thereof or its contractors or subcontractors. The views and opinions of authors expressed herein do not necessarily state or reflect those of the United States Government or any agency thereof. 


\begin{tabular}{|l|c|}
\hline \multicolumn{1}{|c|}{$\begin{array}{c}\text { NSLS II TECHNICAL NOTE } \\
\text { BROOKHAVEN NATIONAL LABORATORY }\end{array}$} & NUMBER: 193 \\
\hline AUTHOR $\quad$ S. Seletskiy \& T. Shaftan & $9 / 24 / 2015$ \\
\hline $\begin{array}{l}\text { TITLE } \\
\text { Reviewed approach to defining the Active Interlock Envelope for Front } \\
\text { End ray tracing }\end{array}$ & \\
\hline
\end{tabular}

BNL-108464-2015-I R

\title{
Reviewed approach to defining the Active Interlock Envelope for Front End ray tracing
}

\author{
S. Seletskiy, T. Shaftan \\ September 24, 2015
}

\section{Introduction}

To protect the NSLS-II Storage Ring (SR) components from damage from synchrotron radiation produced by insertion devices (IDs) the Active Interlock (AI) keeps electron beam within some safe envelope (a.k.a Active Interlock Envelope or AIE) in the transverse phase space [1]. The beamline Front Ends (FEs) are designed under assumption that above certain beam current (typically $2 \mathrm{~mA}$ ) the ID synchrotron radiation (IDSR) fan is produced by the interlocked e-beam. These assumptions also define how the ray tracing for FE is done.

To simplify the FE ray tracing for typical uncanted ID it was decided to provide the Mechanical Engineering group with a single set of numbers ( $\left.\mathrm{x}, \mathrm{x}^{\prime}, \mathrm{y}, \mathrm{y}^{\prime}\right)$ for the AIE at the center of the long (or short) ID straight section. Such unified approach to the design of the beamline Front Ends will accelerate the design process and save valuable human resources.

In this paper we describe our new approach to defining the AI envelope and provide the resulting numbers required for design of the typical Front End.

\section{General Considerations}

Two main features of our approach to finding proper AIE are back ray tracing (BRT) and treating each ID straight section independently. Under this approach we consider each limiting aperture in the SR vacuum chamber that the IDSR fan from individual ID can potentially touch and perform BRT for this aperture. From simple geometric considerations (see Fig. 1) we find respective No-Touch Envelope (NTE) for the electron beam. When e-beam is positioned within the NTE the IDSR fan does not touch the aperture. Apparently, one must define some reasonable cut off limits for the tales of the IDSR fan. Later on we will discuss our choice for these limits.

It is easy to see that for the asymmetric horizontal aperture shown in Fig. 1 located at distance $S$ from the observation point in vacuum chamber with aperture +/-A the NTE is given by:

$$
\begin{aligned}
& x^{\prime} \equiv \alpha_{p}=\frac{a_{p}-x}{S} \\
& x^{\prime} \equiv \alpha_{n}=-\frac{a_{n}+x}{S} \\
& x \in[-A, A],
\end{aligned}
$$

So far we treated the IDSR as a beam with zero divergence rather than a fan. To take into account the angular divergence $2 \alpha_{\text {div }}$ of actual IDSR fan one has to substitute $\alpha_{p}$ and $\alpha_{n}$ in Eq. 
(1) with $\alpha_{p}-\alpha_{d i v}$ and $\alpha_{n}+\alpha_{d i v}$. To take into account possible relative misalignment $+/-m$ of various SR components one shall substitute $a_{p}$ and $a_{n}$ in Eq. (1) with $a_{p}-m$ and $a_{n}-m$.
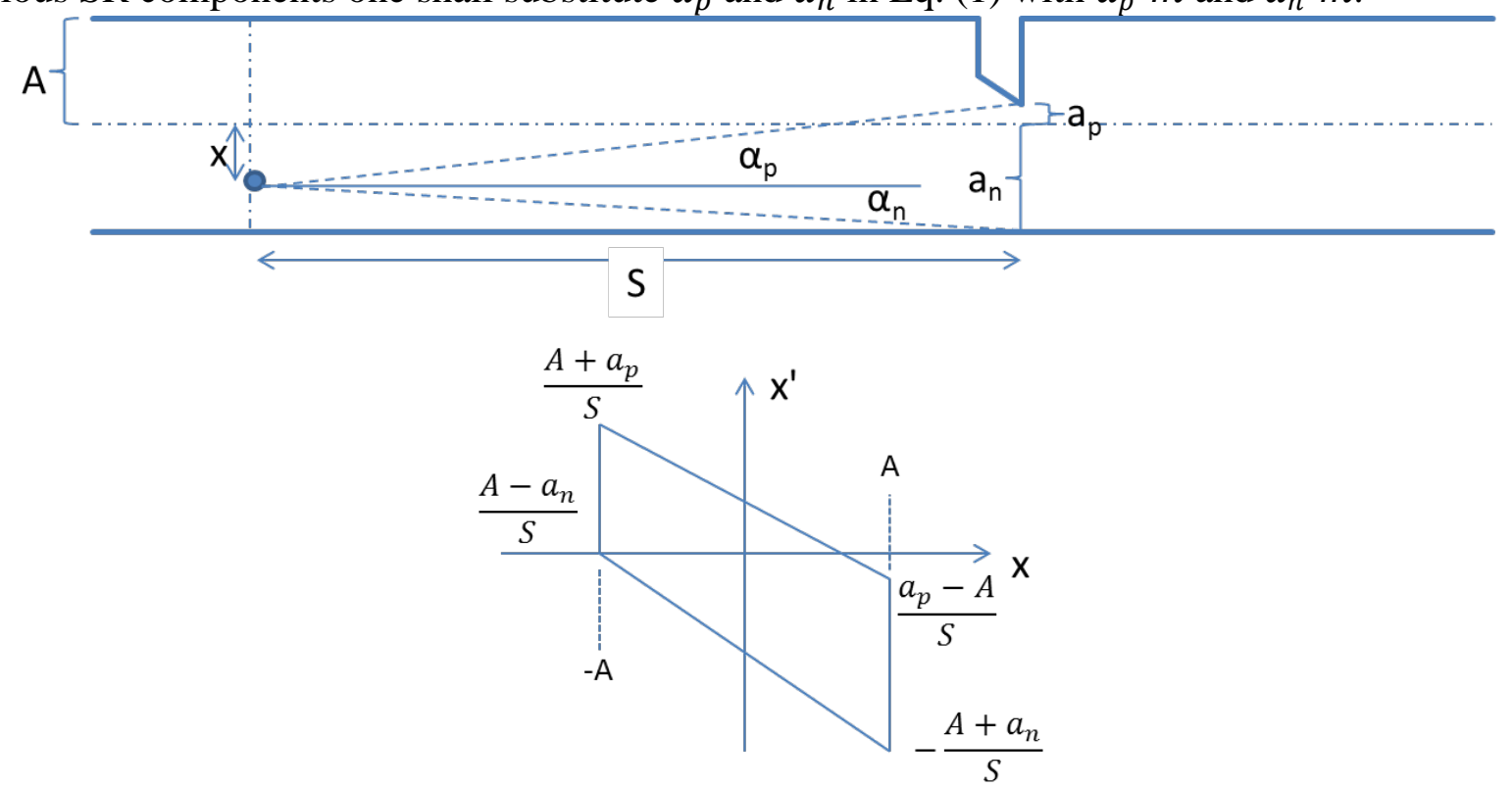

Figure 1: The top plot schematically shows the geometry of limiting horizontal aperture located at distance $S$ downstream of observation point. The bottom plot shows NTE at observation point.

Following the described procedure we define the NTE for each limiting aperture for particular ID and then overlap found NTEs. In case the ID center does not coincide with the center of respective straight section we map obtained NTE to the center of the ID straight. As a result we define the "global" NTE that protects all limiting apertures from IDSR. The described algorithm of finding NTE is schematically shown in Fig. 2.

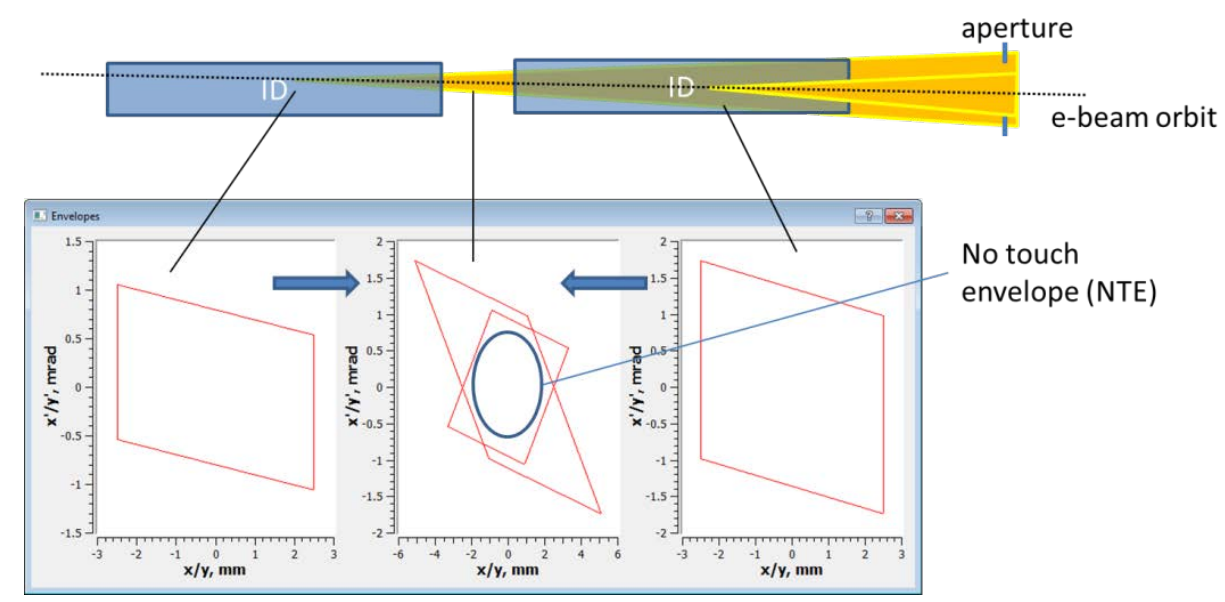

Figure 2: Finding "global" NTE at the center of the straight section with two IDs and one limiting aperture.

\section{Power Density from Planar Undulator}

To find the power density of IDSR in case of planar undulator or wiggler of arbitrary $K$ we adopt an approach from [2]. The angular power density of the synchrotron radiation from planar undulator with deflection parameter $K$ is:

$$
\begin{gathered}
P(\theta, \psi)=\frac{6 P_{T} \gamma^{2}}{(\pi K)^{2}} \cdot \int_{b-K}^{b+K} \sqrt{K-(b-y)^{2}} \frac{a^{2}+(2 a-4) y^{2}+y^{4}}{\left(a+y^{2}\right)^{5}} d y \\
\begin{array}{c}
a=1+(\gamma \psi)^{2} \\
b=\gamma \theta
\end{array} \\
P_{T}=0.633 L I E^{2} B^{2},
\end{gathered}
$$

Here $L$ is an undulator length, $I$ is the beam current, $E$ is the beam energy, $B$ is magnetic field 
of the undulator, $\gamma$ is relativistic factor and the meaning of angles $\theta, \psi$ is shown in Fig. 3 .

\section{Coordinate system}

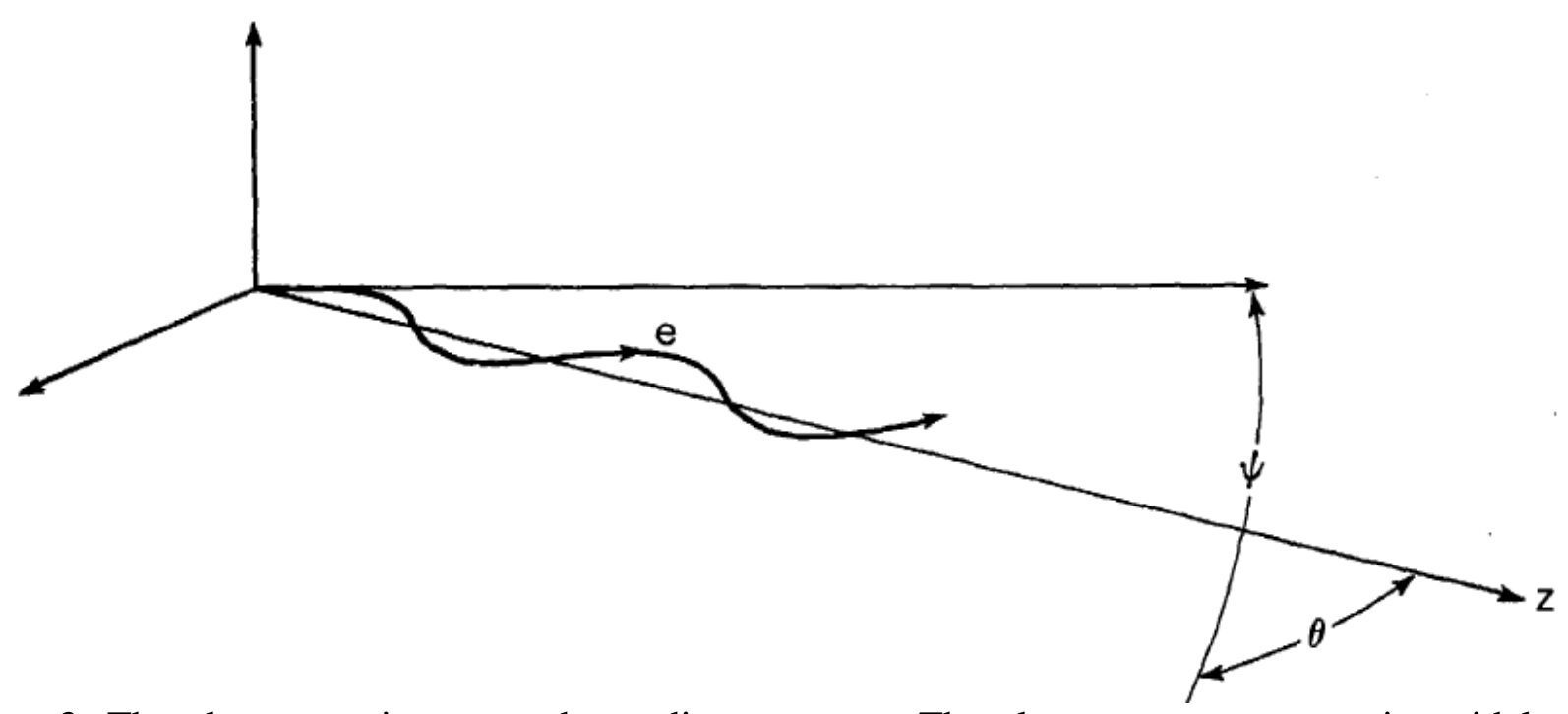

Figure 3: The electron trajectory and coordinate system. The electron moves on a sinusoidal trajectory in horizontal plane $x$-z. $\theta$ and $\psi$ are the angles of observation in the horizontal and vertical directions, respectively.

The expression in (2) is easy to integrate numerically. We use Python "scipy.integrate.quad" function [3] for that purpose. To check the validity of obtained results we benchmarked our code with SRW [4]. Comparison of numerical integration of Eq. (2) with SRW calculations shows good agreement of obtained power density distributions down to at least 1 meter distance from observation point to the source of radiation.

Plots in Fig. 4 show an example of such comparison performed for one of the proposed NSLSII IDs (cell 19 LAX ID). LAX ID length is $1 \mathrm{~m}$, its period is $25 \mathrm{~mm}$, its peak magnetic field is $0.94 \mathrm{~T}$ and $\mathrm{K}$ is 2.2 .

We conclude from our comparison that the precision of analytic formula is remarkably good. The worst discrepancy that we observed was $\sim 0.5 \%$ difference between peak (on-axis) power densities calculated by SRW and from Eq. (2). Such calculation precision is more than enough for the design of IDSR protection.
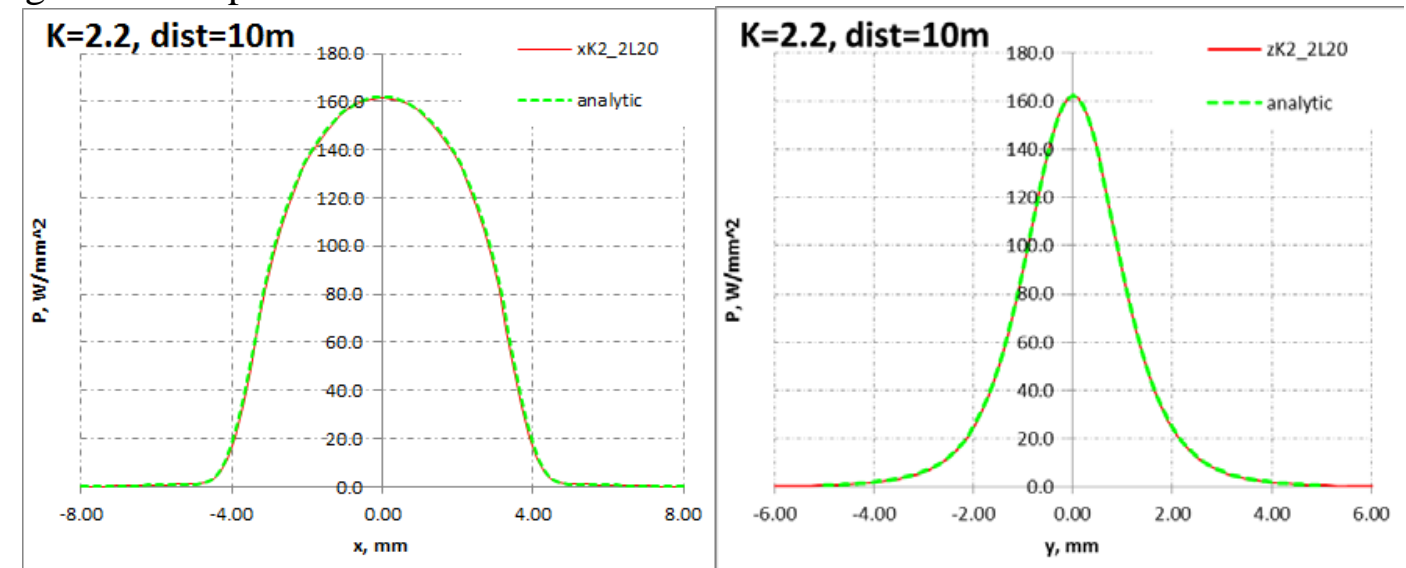

a.) 


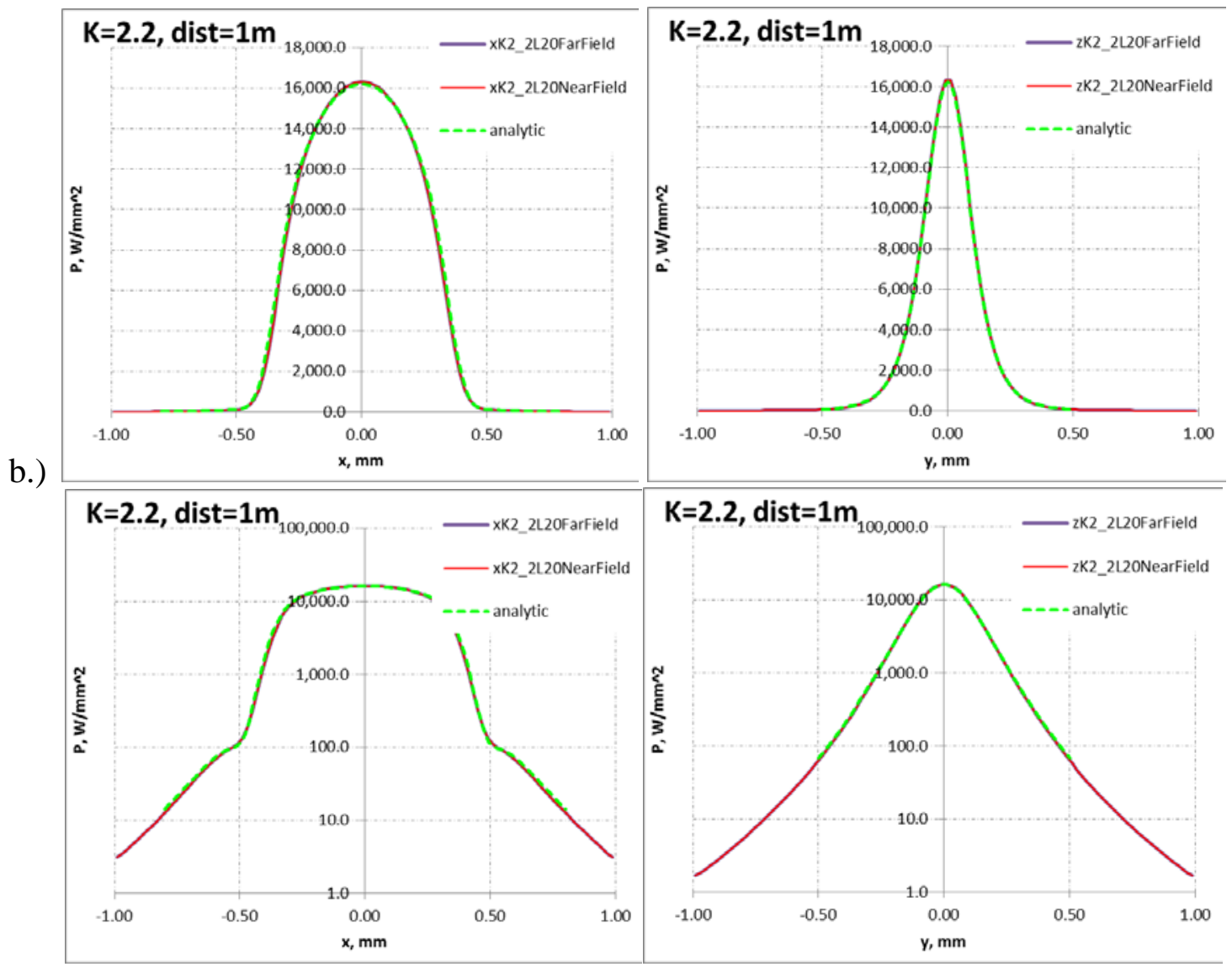

Figure 4: Comparison of numerically integrated Eq. 2 with SRW calculations. Plots (a) show horizontal and vertical power density distribution at $10 \mathrm{~m}$ distance from the undulator. Red solid line represents SRW results, green dotted line is analytic formula (2). Plots (b) show power density distribution at $1 \mathrm{~m}$ distance from the undulator. Blue and red solid lines give SRW results in far field and near field approximations respectively. Green dotted line shows analytic results. Two top plots (b) are given in linear scale; two bottom plots show power density in logarithmic scale demonstrating agreement between SRW and analytic calculations down to $0.1 \%$ of peak power density.

It is worth to mention that in SRW we used exact e-beam parameters. Thus, we conclude that due to the small beam emittance a "single particle" Eq. (2) can be applied to NSLS-II IDs without any farther modifications.

To calculate the IDSR power load on various surfaces along the path of the photon beam one has to convert $P(\theta, \psi)$ to $P(x, y)$ by substitution: $x=\theta \cdot S, y=\psi \cdot S$, where $S$ is the distance from the ID to the surface of interest. In most general case the power deposited on the surface is:

$$
P_{\text {surf }}=\iint_{X Y_{1}\left(g_{1}, \ldots, g_{n}, \vartheta, \varphi, \Delta x, \Delta y\right)}^{X Y_{2}\left(g_{1}, ., g_{n}, \varphi, \Delta x, \Delta y\right)} P(x, y) d x d y,
$$

where integration is performed in coordinates of the surface intercepting the IDSR fan. In the general case the limits of integration are the functions of parameters $g_{1}, \ldots, g_{n}$ defining the geometry of this surface, of the horizontal and vertical angles $\vartheta, \varphi$ representing the inclination of the surface with respect to the IDSR fan and of the horizontal and vertical displacements $\Delta x, \Delta y$ of this surface with respect to the reference frame of the photon beam.

\section{Finding AIE}

Prior to applying the ideas outlined above to finding the AIE for typical ID we must point to the following. From accelerator physics point of view it is reasonable to define the AIE as a diamond shape in the transverse phase space. The ID BPMs are usually located symmetrically on both sides of the center of the straight section. Therefore, interlocking the beam in these IDs within some limits provides the diamond shape interlock at the center of ID straight. On the other 
hand, the diamond shape is not very useful for FE ray tracing since ray tracings done using four corners of a diamond phase-space can lead to unsafe conditions downstream of the first beam intercepting element (DW absorber, ID absorber or fixed mask). It is important to use rectangular AI envelope for ray tracing because it is sufficient to use only the four corner points of a rectangular phase space to unambiguously define the apertures of FE components.

The typical NSLS-II ID is a $3 \mathrm{~m}$ long planar undulator with horizontal polarization centered in the straight section. The vertical divergence of IDSR fan for such devices does not depend on their deflection parameters. For our calculations we choose angle at which power density is $1 \%$ of peak on-axis power density of IDSR. This gives $+/-0.41 \mathrm{mrad}$ for vertical angular divergence of the fan.

The horizontal divergence of the IDSR fan varies depending on the ID parameters. We choose to perform calculations with $+/-1$ mrad horizontal angular divergence. Such divergence is factor of 2 higher than divergences of any existing or planned NSLS-II IDs with exception of damping wigglers, which are not the topic of this paper.

The horizontal and vertical limiting apertures downstream of both long and short straight sections as well as their distances from the center of the straight section are given in Table 1. Most of the limiting apertures in the table have self-explanatory names. Probably the only one that requires additional explanation is the vertical aperture of the dipole chamber.

As the IDSR travels through the storage ring it sees the transition from a regular vacuum chamber to the antechamber. For IDSR this transition is seen at an angle, thus creating a long tapering from $25 \mathrm{~mm}$ to $15 \mathrm{~mm}$.

\begin{tabular}{|l|r|r|r|r|r|}
\hline \multicolumn{7}{|c|}{ Long Straight } \\
\hline Limiting apertures & \multicolumn{1}{|c|}{$\mathrm{m}$} & $\mathrm{Xp}, \mathrm{mm}$ & $\mathrm{Xn}, \mathrm{mm}$ & $\mathrm{Yp}, \mathrm{mm}$ & \multicolumn{1}{|c|}{$\mathrm{rn}, \mathrm{mm}$} \\
\hline Photon absorber & 1.62 & 25 & 38 & 12.5 & 12.5 \\
\hline 5" RF bellows & 4.1595 & 38 & 38 & 12.5 & 12.5 \\
\hline Stick absorber & 7.8163 & 25 & 38 & 10.5 & 10.5 \\
\hline 6" RF bellows & 8.1294 & 38 & 38 & 12.5 & 12.5 \\
\hline Dipole chamber & 10.5904 & & - & 7.5 & 7.5 \\
\hline Crotch absorber & 11.0482 & 35 & 40 & & - \\
\hline Undulator absorber & 11.4414 & 15 & 15 & 15 & 15 \\
\hline
\end{tabular}

\begin{tabular}{|l|r|r|r|r|r|}
\hline \multicolumn{7}{|c|}{ Short Straight } \\
\hline Limiting apertures & Z, $\mathrm{m}$ & \multicolumn{1}{|l|}{ Xp, $\mathrm{mm}$} & $\mathrm{Xn}, \mathrm{mm}$ & $\mathrm{Yp}, \mathrm{mm}$ & \multicolumn{1}{l}{ Yn, $\mathrm{mm}$} \\
\hline Photon absorber & 1.882 & 25 & 38 & 12.5 & 12.5 \\
\hline 5" RF bellows & 2.9967 & 38 & 38 & 12.5 & 12.5 \\
\hline Stick absorber & 6.8895 & 25 & 38 & 10.5 & 10.5 \\
\hline 6" RF bellows & 7.0503 & 38 & 38 & 12.5 & 12.5 \\
\hline Dipole chamber & 9.34909 & & - & 7.5 & 7.5 \\
\hline Crotch absorber & 9.8069 & 35 & 40 & & - \\
\hline
\end{tabular}

Table 1: Limiting apertures downstream of long and short straight sections.

Using described parameters we perform the BRT for both long and short straight section. The tracing is done automatically with a dedicated Python-based code.

Figure 5 shows tracing results in horizontal plane for a long straight section. 


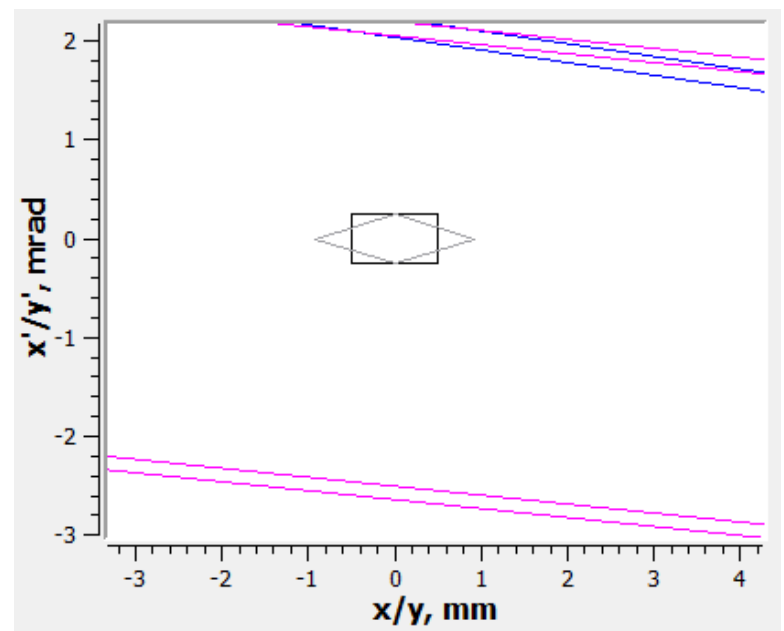

Figure 5: Horizontal BRT for Long straight section. The black rectangle shows $+/-0.5 \mathrm{~mm}$, $+/ 0.25$ mrad envelope. Grey diamond shows the respective +/-0.25 mrad diamond shape envelope and is given for the reference.

The NTE is rather large in horizontal direction. It easily accommodates rectangular $+/-0.5 \mathrm{~mm}$, +/-0.25 mrad AIE. Of course, one can define much larger horizontal AIE in horizontal plane but this will complicate design of respective FE.

BRT results in long straight section for vertical plane are shown in Fig. 6.

The rectangular $+/-0.25 \mathrm{mrad}+/-0.5 \mathrm{~mm}$ envelope is larger than vertical plane NTE for the long straight section. The e-beam located either at lower left or at upper right corners of such AIE will generate IDSR fan that will be depositing some power on dipole chamber. There are a few possible solutions to this problem.

First of all, such problem was considered for already installed and commissioned NSLS-II IDs. It was found that for typical ID the deposited power on the dipole chamber does not cause temperature increase higher than to $100 \mathrm{C}$ for angular e-beam deflections of up to $0.5 \mathrm{mrad}$ [5].

Second, reducing the rectangular AIE to +/-0.2 mrad or switching from rectangular to diamond shape AIE can eliminate the problem altogether as Fig. 6 demonstrates.

Therefore, we suggest utilizing +/-0.25 mrad +/-0.5 mm AIE for the purpose of design of the typical FE. For any particular ID in the long straight section we will perform additional analysis to finalize the actual AIE in that section.
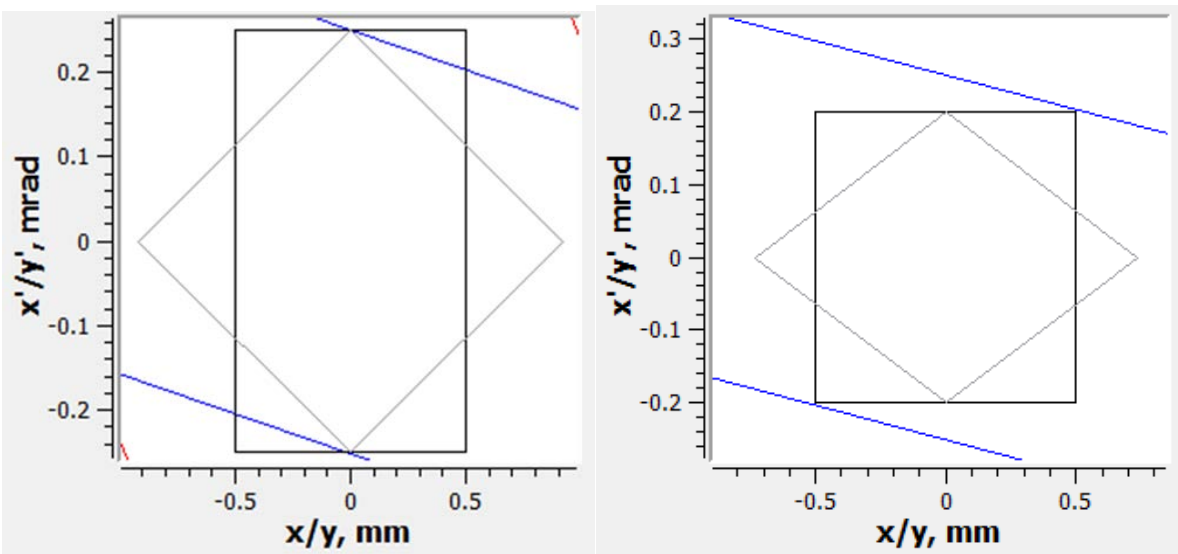

Figure 6: Vertical BRT for the long straight section. Blue lines show borders of dipole chamber NTE. The black rectangle shows the AIE. Left plot shows $+/-0.5 \mathrm{~mm},+/ 0.25$ mrad envelope and right plot shows $+/-0.5 \mathrm{~mm},+/-0.2$ mrad envelope. Grey shapes show the respective diamond envelopes for each rectangular AIE.

The same studies were performed for horizontal and vertical AIE in short straight section. Respective results are shown in Fig. 7 and Fig. 8. 


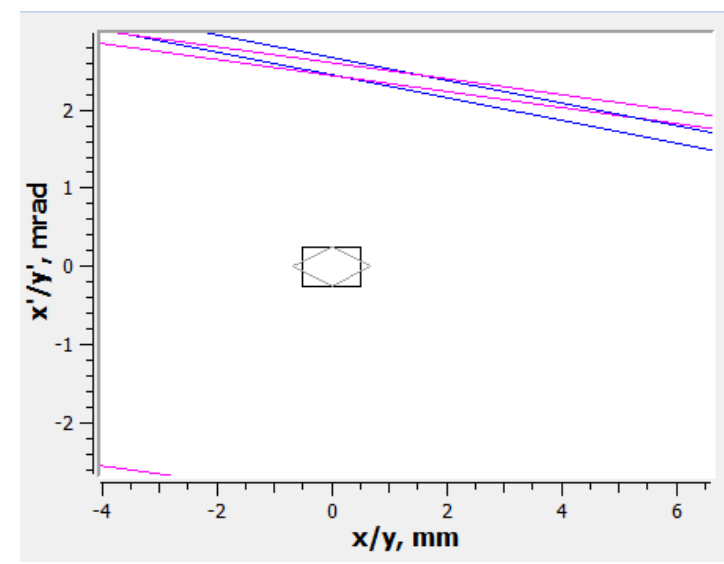

Figure 7: Horizontal BRT for short straight section. The black rectangle shows +/-0.5 mm, +/0.25 mrad envelope. Respective diamond envelope is shown in grey color.

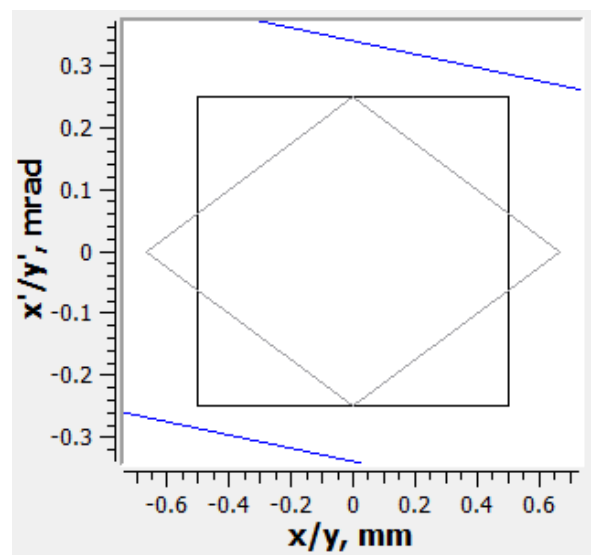

Figure 8: Vertical BRT for short straight section. The black rectangle shows $+/-0.5 \mathrm{~mm},+/-0.25$ mrad envelope. Respective diamond envelope is shown in grey color.

As one can see for the short straight section both horizontal and vertical NTEs are large enough to accommodate rectangular $+/-0.5 \mathrm{~mm},+/-0.25 \mathrm{mrad}$ envelope.

\section{Is the found AIE size large enough?}

In this section we follow the ideas presented in [7]. To estimate whether the particular AIE size is compatible with high efficiency operations we estimate how many beam dumps one can expect due to the violation of given AIE limits.

We will consider only the effect of corrector failure on the beam orbit. The reason for that is that the quadrupole or dipole failure will dump the beam regardless of whether the beam is interlocked or not. The effect of sextupole failure on beam orbit is negligible since the beam is well centered through the multipoles. As for the BPM failure it will cause the trip regardless of the AIE size.

Typical distribution of the strength of storage ring correctors [8] is shown in Fig. 9
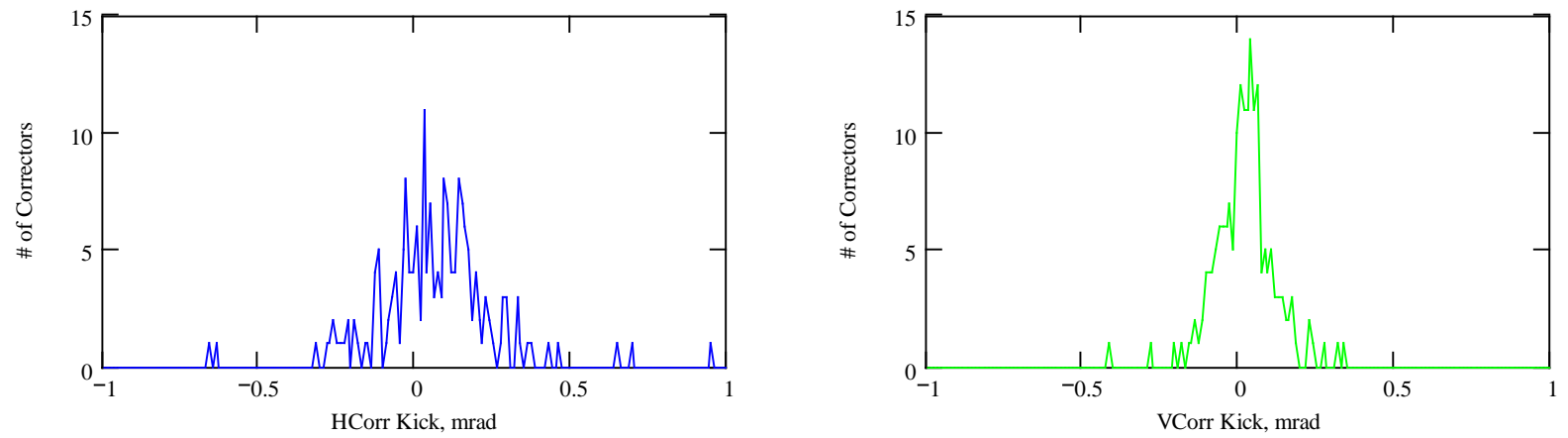

Figure 9: Typical distribution of the kicks of horizontal and vertical correctors in NSLS-II 
storage ring.

The beam deflection at the center of ID $j$ due to the change $\theta_{i}$ in the kick of corrector $i$ is:

$$
x_{j}=\frac{\sqrt{\beta_{j} \beta_{i}}}{2 \sin (\pi v)} \theta_{i} \cos \left(\left|\varphi_{j}-\varphi_{i}\right|-\pi v\right),
$$

The correctors are evenly spread around the ring and eventually the insertion devices will be installed in almost every straight section. Therefore, it is reasonable to consider $\pi$ phase advance between the ID and the corrector in (4). The average $\beta$-function is $\sim 11.8 \mathrm{~m}$ horizontally and $\sim 14.3 \mathrm{~m}$ vertically. Horizontal $\beta$-function at the center of the long straight section is $\sim 19.6 \mathrm{~m}$ and vertical $\beta$-function is $\sim 3.4 \mathrm{~m}$. The horizontal and vertical tunes are 33.22 and 16.26 respectively. Plugging these numbers into (4) we find that the fault of the corrector will violate the AIE if its horizontal and vertical kicks are: $\theta_{x}>0.042 \mathrm{mrad}$ and $\theta_{y}>0.105 \mathrm{mrad}$.

Our experience shows that the average number of correctors' failures during one year of operation is $N_{F} \approx 16$ [9].

The number of correctors' failures causing beam trip due to AIE violation can be estimated as:

$$
N_{\text {AIEtrip }}=N_{F} \frac{N_{\theta>\theta \max }}{N_{C}} \text {, }
$$

Here $N_{C}=180$ is the total number of correctors and $N_{\theta>\theta \max }$ is the number of correctors, which kick exceeds the kicks estimated from (4). In horizontal plane $N_{\theta>\theta \max }$ is 137 and in vertical plane $N_{\theta>\theta \max }=39$. Substituting these numbers into (5) we find that the expected number of beam dumps caused by AIE violation is 12.2 and 3.5 for horizontal and vertical correctors' trips respectively.

Our experience shows that recovery from the AI trips usually takes less than $20 \mathrm{~min}$. The yearly operation time for NSLS-II [7] is $5000 \mathrm{~h}$. Thus, we conclude that for the given horizontal and vertical AIEs the reduction in machine availability is expected to be $0.07 \%$ and $0.02 \%$ respectively. Since the target availability of NSLS-II is 98.5\%, the obtained availability reduction seems to be tolerable.

\section{Some additional considerations}

The goal of this paper is to define the AIE for design of a typical FE. We suggest using rectangular $+/-0.5 \mathrm{~mm},+/-0.25$ mrad envelope for the FE ray tracing in both long and short straight sections. Yet, this doesn't mean that we will necessarily use such AIE for all typical IDs in our AI system.

As an example, we might decide to switch to diamond envelope or to smaller rectangular envelope in vertical plane for long straight section, as was discussed above. We also might eventually decide to use a larger AIE where it is allowed. Our experience proves that the analysis of several already designed, installed and commissioned FEs frequently shows that NTE for these FEs is much larger than the nominal AIE that was used for their initial design (there is some information on this topic in Ref. [6]).

Of course, any change in actual AIE will be happening only after thorough analysis of both the storage ring components and already designed FE components downstream of the particular ID similar to the analysis described in this paper.

\section{Beam envelope for BM-A, BM-B and 3PW}

It was determined [10] that for the beam deviations inside the two dipoles of a cell (BM-A and BM-B) as well as at 3-pole wiggler (3PW) it will be satisfactory to use an estimate of the beam acceptance (BA) at their respective locations rather than defining the precise AIE for these devices.

The BAs for BM and 3PW were found in [11] for the approximation of linear lattice. Such approximation overestimates the BAs as compared to the BAs in real storage ring. In addition to that, for the purpose of $\mathrm{FE}$ design we suggest using the rectangular phase space area (for explanations see section "Finding AIE") including the defined BA, rather than the actual BA. Thus, the usage of proposed envelopes guarantees that the position of the real beam in the phase 
space cannot exceed the limits of such envelope.

Applying an outlined procedure we obtain the following results:

\begin{tabular}{|l|l|l|l|l|}
\hline & $\mathrm{x}, \mathrm{mm}$ & $\mathrm{x}^{\prime}, \mathrm{mrad}$ & $\mathrm{y}, \mathrm{mm}$ & $\mathrm{y}^{\prime}, \mathrm{mrad}$ \\
\hline BM-A in even cell & $+/-12$ & $+/-10$ & $+/-12.5$ & $+/-2.5$ \\
\hline BM-B in even cell & $+/-11$ & $+/-10$ & $+/-12$ & $+/-2.2$ \\
\hline BM-A in odd cell & $+/-12$ & $+/-10$ & $+/-12.5$ & $+/-2.5$ \\
\hline BM-B in odd cell & $+/-11$ & $+/-10$ & $+/-12$ & $+/-2.2$ \\
\hline 3PW in even cell & $+/-10$ & $+/-17$ & $+/-12$ & $+/-2.5$ \\
\hline 3PW in odd cell & $+/-10$ & $+/-17$ & $+/-12$ & $+/-2.5$ \\
\hline
\end{tabular}

Table 2: Beam acceptance envelopes defined for BM-A, BM-B and 3PW.

\section{Conclusion}

In this paper we derived the Active Interlock envelope for the electron beam for typical ID installed either in long or short straight section.

We suggest that for design of the typical beamline Front End one shall use the following Active Interlock envelope:

Rectangular envelope of +/-0.5 mm and +/-0.25 mrad.

Such envelope shall be used both for short and long straight sections both in horizontal and vertical planes.

In the future, we might change the actual AIE based on the analysis of each particular ID and of the already designed FE.

The envelopes that one has to use for the beam deviations at BM-A, BM-B and $3 P W$ are defined in Table 2.

\section{References}

[1] S. Seletskiy et al., Commissioning of Active Interlock System for NSLS II Storage Ring, IPAC15 proceedings, 2015.

[2] K.J. Kim, Angular distribution of undulator power for an arbitrary deflection parameter K, LBL-20167, 1985.

[3] Scipy Reference Guide, http://docs.scipy.org/doc/scipy/reference/

[4] O. Chubar, P. Elleaume, Accurate and Efficient Computation of Synchrotron Radiation in the Near Field Region, EPAC98 proceedings, 1998.

[5] V. Ravindranath, Dipole Chamber FEA-Active interlock envelope for IVU beam, Presentation, 4/26/2011.

[6] S. Seletskiy, C. Amundsen, K. Ha, A. Hussain, Safe operating conditions for NSLS-II Storage Ring Frontends commissioning, BNL-107813-2015-IR, 2015.

[7] J. Choi, F. Willeke, Nuclear Instruments and Methods A 681 (2012) 1-6.

[8] Live data taken on June 24, 2015 during the users run.

[9] Private communications with Reid Smith.

[10] Private communications with Sushil Sharma and Mechanical Engineering Group.

[11] I. Pinayev, Orbit Envelopes for NSLS-II Ring Synchrotron Radiation Sources. 